\title{
Evolution of coherence singularities of Schell-model beams
}

\author{
José A. Rodrigo and Tatiana Alieva*1 \\ ${ }^{1}$ Universidad Complutense de Madrid, Facultad de Ciencias Físicas, Ciudad Universitaria s/n, Madrid 28040, Spain \\ *Corresponding author: talieva@fis.ucm.es
}

Compiled July 10, 2015

We show that the propagation of the widely used Schell-model partially coherent light can be easily understood using the ambiguity function. This approach is especially beneficial for the analysis of the mutual intensity of Schell-model beams (SMBs), which are associated with stable coherent beams such as Laguerre-, Hermite-, and Ince-Gaussian. We study the evolution of the coherence singularities during the SMB propagation. It is demonstrated that the distance of singularity formation depends on the coherence degree of the input beam. Moreover it is proved that the shape, position and number of singularity curves in far field are defined by the associated coherent beam. () 2015 Optical Society of America

OCIS codes: (030.0030) Coherence and statistical optics; (260.6042) Singular optics; (070.2575) Fractional Fourier transforms

\section{http://dx.doi.org/10.1364/ol.XX.XXXXXX}

Singular optics was born in the context of coherent light [1] and its applications in optical particle manipulation, imaging and free-space communications are widely acknowledged. Later, the existence of spatial correlation singularities has been found [2-4]. A peculiarity of the partially coherent beam singularities is that the intensity in such points has not vanished as it must occur for the coherent case. In particular, it has been reported [5-7] that the spatial coherence function of Schell model Laguerre-Gaussian (LG) beam has robust circular phase singularities in far field even when its intensity distribution is almost Gaussian. Such hidden singularities might play an important role, for example, for information encoding in the context of free-space communications. To exploit them, however, a better understanding of the singularity nature is required. In this letter we analyze the formation and evolution of singularities in the coherence function during propagation of Schell-model beams (SMBs). These singularities are associated with cross-correlation function zero points which usually correspond to the transition between positively and negatively correlated field and are experimentally observed as dislocations of the interference fringes. First of all, we demonstrate the advantage of using the ambiguity function (AF) for comprehensive description of the SMB propagation. Then we explain the splitting of the coherence phase singularities during the beam propagation and show that their stability depends on the stability of the coherent beam associated with the SMB. We remind that a stable coherent beam does not change the form of its intensity distribution apart from a scaling during propagation. Stability of coherence singularities means that there exist a direct relation between the number and position of the coherence singularities in the input and far-field planes. As an example, the evolution of coherence singularities of the Hermite-Gaussian (HG) SMBs is considered.

In general, the analysis of partially coherent beams is much more difficult than the coherent one. Its description even in scalar quasi-monochromatic case involves complex-valued 4D function, i.e. mutual intensity (MI) defined as $\Gamma\left(\mathbf{r}_{1}, \mathbf{r}_{2}\right)=$ $\left\langle f\left(\mathbf{r}_{1}\right) f^{*}\left(\mathbf{r}_{2}\right)\right\rangle$ [8]. Here, $f(\cdot)$ is a complex field amplitude and $\mathbf{r}^{t}=(x, y)$ is a position vector at the plane transverse to the beam propagation direction $z$. In the latter expressions, $t$ stands for the transpose operation and $\langle\cdot\rangle$ stands for ensemble averaging, which is omitted for the coherent case: $\Gamma_{c}\left(\mathbf{r}_{1}, \mathbf{r}_{2}\right)=f\left(\mathbf{r}_{1}\right) f^{*}\left(\mathbf{r}_{2}\right)$. Partially coherent beams are often assumed to be SMBs [9], whose MI is given in the following simplified form

$$
\Gamma\left(\mathbf{r}_{1}, \mathbf{r}_{2}\right)=\Gamma_{\mathcal{C}}\left(\mathbf{r}_{1}, \mathbf{r}_{2}\right) \gamma\left(\mathbf{r}_{1}-\mathbf{r}_{2}\right)
$$

with $\gamma(\cdot)$ being an equal-time complex degree of spatial coherence (DoC). A SMB is generated, for example in microscopy, when a partially coherent plane wave characterized by $\gamma(\cdot)$ propagates through a sample described by a complex transmittance function $f(\mathbf{r})$. Note that the intensity distribution of the SMBs, $I(\mathbf{r})=\Gamma(\mathbf{r}, \mathbf{r})=\Gamma_{\mathcal{c}}(\mathbf{r}, \mathbf{r})=|f(\mathbf{r})|^{2}$, does not depends of $\gamma(\cdot)$ in the input plane. Nevertheless, this relation as well as the Eq. (1) are only valid for the input plane and become much more complex for a propagating beam. Let us consider the propagation of the SMB through a paraxial system described by the $4 \times 4$ ray transformation matrix

$$
\mathbf{T}=\left[\begin{array}{ll}
\mathbf{A} & \mathbf{B} \\
\mathbf{C} & \mathrm{D}
\end{array}\right]
$$

which includes the case of a free space interval for $\mathbf{D}=\mathbf{A}=\mathbf{I}$ and $\mathbf{C}=\mathbf{0}$ (where $\mathbf{I}$ is a unity matrix $2 \times 2$ ). The propagation can be easily described by using the $\mathrm{AF}, A(\mathbf{r}, \mathbf{p})$, which is a complex function related to the MI via the Fourier transform (FT):

$$
A(\mathbf{r}, \mathbf{p})=\iint \Gamma\left(\mathbf{R}+\frac{\mathbf{r}}{2}, \mathbf{R}-\frac{\mathbf{r}}{\mathbf{2}}\right) \exp [-i 2 \pi \mathbf{R} \mathbf{p}] \mathrm{d} \mathbf{R} .
$$


Here and further we use normalized coordinates $s^{-1} \mathbf{r}=: \mathbf{r}$, $s \mathbf{p}=: \mathbf{p}$, where $s$ has dimension of length and therefore all the variables as well as the parameters of the ray transformation matrix $\mathbf{T}$ are dimensionless. In the input plane one obtains a simple relation between the AF of a SMB and the AF for the associated coherent beam, denoted by $A_{\mathcal{c}}(\mathbf{r}, \mathbf{p})$ :

$$
\begin{aligned}
A(\mathbf{r}, \mathbf{p}) & =\gamma(\mathbf{r}) \iint \Gamma_{\mathcal{C}}\left(\mathbf{R}+\frac{\mathbf{r}}{2}, \mathbf{R}-\frac{\mathbf{r}}{\mathbf{2}}\right) \exp [-i 2 \pi \mathbf{R} \mathbf{p}] \mathrm{d} \mathbf{R} \\
& =\gamma(\mathbf{r}) A_{\mathcal{C}}(\mathbf{r}, \mathbf{p}) .
\end{aligned}
$$

The beam propagation through the optical system described by the matrix $\mathbf{T}$ results in an affine transformation of the AF [10]:

$$
A(\mathbf{r}, \mathbf{p} ; \mathbf{T})=A\left(\mathbf{D}^{\mathrm{t}} \mathbf{r}-\mathbf{B}^{\mathbf{t}} \mathbf{p},-\mathbf{C}^{\mathrm{t}} \mathbf{r}+\mathbf{A}^{\mathbf{t}} \mathbf{p}\right) .
$$

Therefore, taking into account the last three equations the MI at the output plane of the system is written as

$$
\begin{aligned}
\Gamma\left(\mathbf{R}+\frac{\mathbf{r}}{2},\right. & \left.\mathbf{R}-\frac{\mathbf{r}}{2} ; \mathbf{T}\right)=\iint \gamma\left(\mathbf{D}^{\mathrm{t}} \mathbf{r}-\mathbf{B}^{\mathrm{t}} \mathbf{p}\right) \\
& \times A_{\mathcal{c}}\left(\mathbf{D}^{\mathrm{t}} \mathbf{r}-\mathbf{B}^{\mathrm{t}} \mathbf{p},-\mathbf{C}^{\mathrm{t}} \mathbf{r}+\mathbf{A}^{\mathrm{t}} \mathbf{p}\right) \exp [i 2 \pi \mathbf{R} \mathbf{p}] \mathrm{d} \mathbf{p} .
\end{aligned}
$$

The analysis of the MI is often reduced to the consideration of the intensity distribution, $I(\mathbf{r})=\Gamma(\mathbf{r}, \mathbf{r})$, and the cross-correlation function (CCF): $\Gamma(\mathbf{r},-\mathbf{r})$. The Eq. (6) is easily particularized for the intensity distribution

$$
I(\mathbf{R} ; \mathbf{T})=\iint A_{c}\left(-\mathbf{B}^{\mathbf{t}} \mathbf{p}, \mathbf{A}^{\mathbf{t}} \mathbf{p}\right) \gamma\left(-\mathbf{B}^{\mathbf{t}} \mathbf{p}\right) \exp [i 2 \pi \mathbf{R} \mathbf{p}] \mathrm{d} \mathbf{p},
$$

and the $\mathrm{CCF}$

$$
\begin{aligned}
\Gamma\left(\frac{\mathbf{r}}{2},-\frac{\mathbf{r}}{2} ; \mathbf{T}\right) & =\iint A_{c}\left(\mathbf{D}^{\mathrm{t}} \mathbf{r}-\mathbf{B}^{\mathrm{t}} \mathbf{p},-\mathbf{C}^{\mathrm{t}} \mathbf{r}+\mathbf{A}^{\mathrm{t}} \mathbf{p}\right) \\
& \times \gamma\left(\mathbf{D}^{\mathrm{t}} \mathbf{r}-\mathbf{B}^{\mathrm{t}} \mathbf{p}\right) \mathrm{d} \mathbf{p} .
\end{aligned}
$$

Moreover, from Eq. (7) the simple well-known relation between the intensity distributions of the SMB and the associated coherent beam, $I_{\mathcal{C}}(\mathbf{R} ; \mathbf{T})$, is obtained:

$$
I(\mathbf{R} ; \mathbf{T})=\iint I_{\mathcal{C}}\left(\mathbf{R}^{\prime} ; \mathbf{T}\right) \widehat{\gamma}_{B}\left(\mathbf{R}-\mathbf{R}^{\prime}\right) \mathrm{d} \mathbf{R}^{\prime},
$$

where $\widehat{\gamma}_{B}(\mathbf{R})=\iint \gamma\left(\mathbf{B}^{\mathbf{t}} \mathbf{p}\right) \exp [-i 2 \pi \mathbf{R} \mathbf{p}] \mathrm{d} \mathbf{p}$.

The expressions Eq. (6), Eq. (8) and Eq. (9) are especially beneficial for the analysis of the propagation of partially coherent SMBs associated with coherent stable beams such as LG, HG, Ince-Gaussian and many others [11]. Since these beams are eigenfunctions for the fractional FT [12], their AFs do not change during propagation through the fractional FT system characterized by the transformation matrix $\mathbf{T}_{\alpha}$ with $\mathbf{A}=\mathbf{D}=\mathbf{I} \cos \alpha$ and $\mathbf{B}=-\mathbf{C}=\mathbf{I} \sin \alpha$, thus $A_{\mathcal{c}}\left(\mathbf{r}, \mathbf{p} ; \mathbf{T}_{\alpha}\right)=A_{\mathcal{c}}(\mathbf{r}, \mathbf{p})$. Therefore, the expression Eq. (8) is reduced to

$$
\Gamma\left(\frac{\mathbf{r}}{2},-\frac{\mathbf{r}}{2} ; \mathbf{T}_{\alpha}\right)=\iint A_{c}(\mathbf{r}, \mathbf{p}) \gamma(\mathbf{r} \cos \alpha-\mathbf{p} \sin \alpha) \mathrm{d} \mathbf{p} .
$$

This is a crucial equation for the analysis of the CCF singularity evolution. Note, that the fractional FT for $\alpha \in[0, \pi / 2]$ describes in paraxial approximation the free-space propagation of the beam's complex field amplitude, apart from scaling and additional quadratic phase shift [13]. It makes the fractional FT system, see for example [14], appropriate for the analysis of CCF singularities of stable SMBs in the Fresnel and far field ( $\alpha=\pi / 2)$ regions. We recall that the spatial coherence singularities of LG-SMBs have been discussed in [5-7] only in far field.
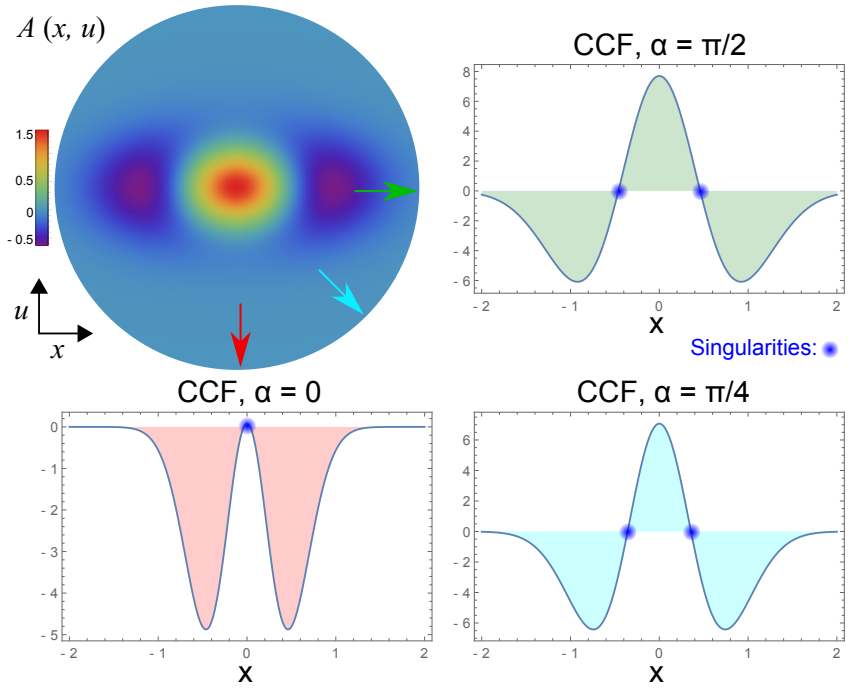

Fig. 1. (Color online) Ambiguity function of the $1 \mathrm{D}$ SMB associated with $\mathrm{HG}$ mode $\mathcal{H}_{1}$ and its projections (AF integration along the directions indicated by arrows) for $\alpha=0, \pi / 4, \pi / 2$ corresponding to the CCF in the input plane, Fresnel and Fraunhofer regions, respectively.

We underline that Eq. (10) allows studying the evolution of the coherence singularities from near- to far-field for SMB related to any stable coherent beam.

From a simple inspection of the CCF of a SMB in the input plane, $\Gamma(\mathbf{r} / 2,-\mathbf{r} / 2)=\gamma(\mathbf{r}) \Gamma_{c}(\mathbf{r} / 2,-\mathbf{r} / 2)$, it is clear that its singularities are defined not only by the singularities of the coherent $\mathrm{CCF}, \Gamma_{\mathcal{C}}(\mathbf{r} / 2,-\mathbf{r} / 2)$, but also by the DoC. For example, the DoC described by sinc or Airy functions adds new singularity points to the SMB CCF. Here we will restrict our analysis to the SMB with Gaussian DoC, $\gamma(\mathbf{r})=\exp \left[-\pi r^{2} / 2 \rho^{2}\right]$, in order to consider the evolution of singularities originated only by the coherent CCF.

As an example, the coherence singularities evolution of the HG-SMB is analyzed. This beam is associated with a coherent HG mode given by $f(\mathbf{r})=\mathcal{H}_{n}(x) \mathcal{H}_{m}(y)$, with

$$
\mathcal{H}_{n}(x)=\frac{2^{1 / 4}}{\sqrt{2^{n} n !}} H_{n}(\sqrt{2 \pi} x) \exp \left[-\pi x^{2}\right],
$$

where $H_{n}(\cdot)$ is an Hermite polynomial of order $n$. For simplicity we have chosen the fundamental Gaussian beam waist equal to $w=s / \sqrt{\pi}$. The MI of the HG-SMB

$$
\begin{aligned}
\Gamma\left(\mathbf{r}_{1}, \mathbf{r}_{2}\right) & =\mathcal{H}_{n}\left(x_{1}\right) \mathcal{H}_{m}\left(y_{1}\right) \mathcal{H}_{n}\left(x_{2}\right) \mathcal{H}_{m}\left(y_{2}\right) \\
\times \exp & {\left[-\pi \frac{\left(x_{1}-x_{2}\right)^{2}+\left(y_{1}-y_{2}\right)^{2}}{2 \rho^{2}}\right] } \\
= & \Gamma_{\mathcal{H}_{n}}\left(x_{1}, x_{2}\right) \Gamma_{\mathcal{H}_{m}}\left(y_{1}, y_{2}\right)
\end{aligned}
$$

is separable in the Cartesian coordinates. The same is true for the $\mathrm{AF}, A_{\mathcal{C}}(\mathbf{r}, \mathbf{p})=A_{\mathcal{H}_{n}}(x, u) A_{\mathcal{H}_{m}}(y, v)$, and the Wigner Distribution (WD) of these beams. The well-known expression for the WD of the HG beam (see for example [15]) and its relation to the AF, $A_{\mathcal{H}_{n}}(x, u)=(-1)^{n} 2^{-1 / 2} W_{\mathcal{H}_{n}}(-x / 2,-u / 2)$, allows for writing the $\mathrm{AF}$ as it follows:

$$
A_{\mathcal{H}_{n}}(x, u)=\sqrt{2} L_{n}\left[\pi\left(x^{2}+u^{2}\right)\right] \exp \left[-\frac{\pi}{2}\left(x^{2}+u^{2}\right)\right],
$$


where $L_{n}(\cdot)$ is a Laguerre polynomial of order $n$. Using the beam separability we first consider the evolution of only one CCF component of the 2D HG-SMB:

$$
\begin{aligned}
& \Gamma_{\mathcal{H}_{n}}\left(\frac{x}{2},-\frac{x}{2} ; \mathbf{T}_{\alpha}\right)= \int A_{\mathcal{H}_{n}}(x, u) \gamma(x \cos \alpha-u \sin \alpha) \mathrm{d} u \\
&=\int \sqrt{2} L_{n}\left[\pi\left(x^{2}+u^{2}\right)\right] \exp \left[-\frac{\pi}{2}\left(x^{2}+u^{2}\right)\right] \\
& \times \exp \left[-\pi \frac{(x \cos \alpha-u \sin \alpha)^{2}}{2 \rho^{2}}\right] \mathrm{d} u .
\end{aligned}
$$

Since $A_{\mathcal{H}_{n}}(x, u)$ is symmetric with respect to rotation in $x-u$ phase space plane the CCF evolution during propagation corresponds to the projection changes of the input AF: $A(x, u)=$ $A_{\mathcal{H}_{n}}(x, u) \exp \left[-\pi x^{2} / 2 \rho^{2}\right]$. This simple tomographic interpretation of the CCF evolution of the SMB beam associated with stable coherent beam is illustrated in Fig. 1 for the case of HG mode with index $n=1$. By integrating $A(x, u)$ over $u$ one obtains the CCF in the input plane $\Gamma_{\mathcal{H}_{n}}(x / 2,-x / 2)$, that corresponds to $\alpha=0$. While by integrating it over $x$, the CCF of the SMB in far field $(\alpha=\pi / 2)$ is calculated. Choosing other integration directions $(\alpha)$ the CCF for Fresnel propagation region is obtained. The integral Eq. (14) can be analytically calculated yielding to the CCF expressed in the form

$$
\begin{aligned}
\Gamma_{\mathcal{H}_{n}}\left(x / 2,-x / 2 ; \mathbf{T}_{\alpha}\right)= & P_{2 n}(\alpha, \rho, x) \exp \left[-\pi x^{2} / 2\right] \\
& \times \exp \left[-\pi x^{2} \frac{\cos ^{2} \alpha}{2\left(\rho^{2}+\sin ^{2} \alpha\right)}\right],
\end{aligned}
$$

where $P_{2 n}(\alpha, \rho, x)$ is a $2 n$-order polynomial of $x$. The polynomial has $2 n$ roots, but they might be complex or multiple. The latter case, for example, corresponds to the case $\alpha=0$ when the number of different roots is reduced to $n$. The zero points of $P_{2 n}(\alpha, \rho, x)$ define the singularity points of the CCF. For example, for $n=1$ two singular points are expressed as

$$
x_{ \pm}= \pm \frac{\sin \alpha \sqrt{\rho^{2}+\sin ^{2} \alpha}}{\sqrt{\pi\left[\rho^{4}+\sin ^{2} \alpha\left(1+2 \rho^{2}\right)\right]}} .
$$

From the analysis of this equation it follows that in the region $\alpha \in(0, \pi / 2]$, associated with free space propagation at the distance $z$ (Fresnel number equals $F=w^{2} / \lambda z=\cot \alpha$, where $\lambda$ is a wavelength), the CCF has two singular points where its sign changes, see blue points in the CCF profiles in Fig. 1. For $\alpha=0$ the two solutions are collapsed. Indeed, in the input plane $(\alpha=0)$ the CCF is given by

$$
\Gamma_{\mathcal{H}_{1}}\left(\frac{x}{2},-\frac{x}{2}\right)=-2 \pi x^{2} \exp \left[-\frac{\pi x^{2}}{2}\right] \exp \left[-\frac{\pi x^{2}}{2 \rho^{2}}\right],
$$

with only one singularity at $x=0$. In this CCF singular point the SMB intensity is also zero. On the other hand, for far field ( $\alpha=\pi / 2)$ the expression Eq. (14) is reduced to

$$
\Gamma_{\mathcal{H}_{1}}\left(\frac{x}{2},-\frac{x}{2} ; \mathbf{T}_{\frac{\pi}{2}}\right)=\frac{2 \rho\left[1-\pi x^{2}\left(\rho^{2}+1\right)\right]}{\left(\rho^{2}+1\right)^{3 / 2}} \exp \left[-\frac{\pi x^{2}}{2}\right],
$$

with two singularity points. Note that the larger $\rho$ corresponds to the smaller interval between these points: $\Delta x=2 / \sqrt{\pi\left(1+\rho^{2}\right)}$, which is zero for the completely coherent case.

The evolution of the CCF and the intensity distribution (calculated using Eq. (9)) for $1 \mathrm{D}$ SMBs associated with $\mathcal{H}_{1}$ and $\mathcal{H}_{2}$
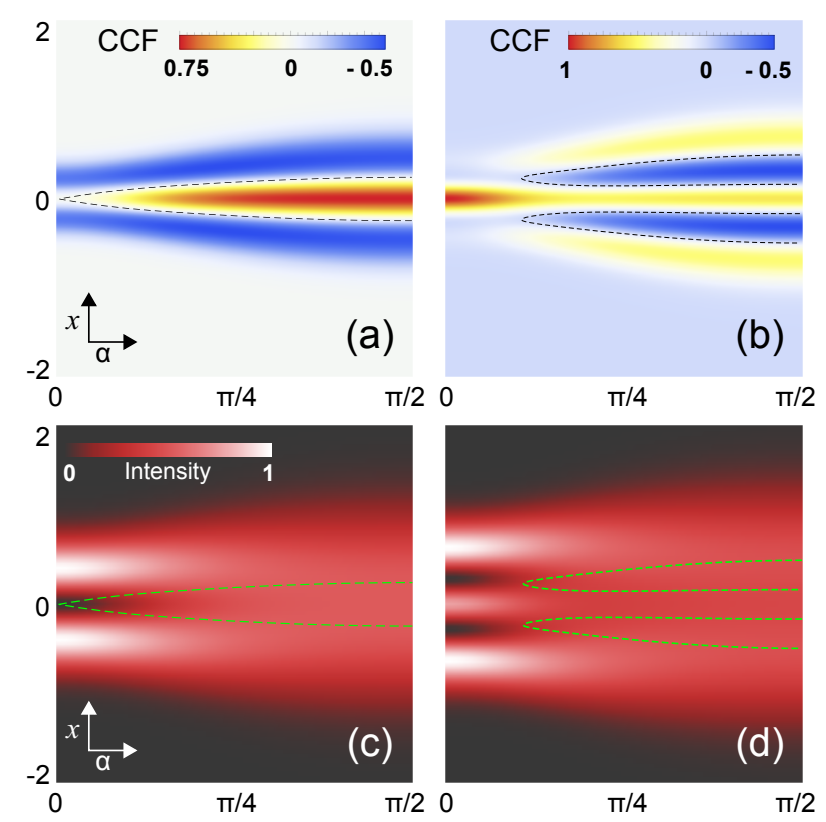

Fig. 2. (Color online) Evolution of the CCF, $\Gamma_{\mathcal{H}_{n}}\left(x,-x ; \mathbf{T}_{\alpha}\right),(\mathrm{a}$, b) and of the intensity distribution $(\mathrm{c}, \mathrm{d})$ during propagation $(\alpha)$ for the 1 D SMBs associated with HG mode $\mathcal{H}_{1}(\mathrm{a}, \mathrm{c})$ and $\mathcal{H}_{2}$ $(b, d)$. Dashed curves indicate singularity points of the CCF.

is shown in Fig. 2. The value $\rho=1 / \sqrt{2}$ chosen in this letter for demonstration corresponds to an input coherence length equal to the fundamental Gaussian beam waist $w$. As it is expected for partially coherent beams the correlation length and the effective beam width increase during the propagation. In Fig. 2(a) and 2(b) one observes that every CCF singular point at the input plane $(\alpha=0)$ is doubled (see dashed lines) in the far field $(\alpha=\pi / 2)$. However, for $\mathcal{H}_{2}-\mathrm{SMB}$ there exists a region of propagation distances ( $\alpha$ from 0 to approximately $\pi / 9$ ) where the CCF does not have any singularity (complex roots for the polynomial $\left.P_{4}(\alpha, \rho, x)\right)$, while for $\mathcal{H}_{1}-\mathrm{SMB}$ two singular points are present for any $\alpha \in(0, \pi / 2]$. Zero intensity points (see Fig. 2(c) and 2(d)) are observed only at the input plane and are washed out during the beam propagation. Note that the intensity values in the CCF singularity points, indicated by green dashed curves in Fig. 2(c) and 2(d), are quite high especially in the far field.

Taking into account these results and the separability of the 2D HG-SMBs, the expression for the evolution of its CCF (and analogously for the intensity distribution) is obtained by multiplication of 2D CCFs: $\Gamma_{\mathcal{H}_{m} \mathcal{H}_{n}}\left(\mathbf{r},-\mathbf{r} ; \mathbf{T}_{\alpha}\right)=$ $\Gamma_{\mathcal{H}_{m}}\left(x,-x ; \mathbf{T}_{\alpha}\right) \Gamma_{\mathcal{H}_{n}}\left(y,-y ; \mathbf{T}_{\alpha}\right)$. In Fig. 3 one can observe how the singularity lines (indicated by dashed lines), which coincide with the position of zero intensity line for $\alpha=0$, change during beam propagation. For $\alpha=\pi / 10$ one horizontal CCF singularity line is transformed into two lines, while the vertical singularity lines disappear. The structure of the HG beam's intensity are washed out in the SMB counterpart for far field, while the CCF doubled singularity-lines displayed in Fig. $3(\alpha=\pi / 2)$ serve as a fingerprint to identify the original coherent beam.

In order to understand the formation process of CCF singularity let us analyze the singularity curves for $1 \mathrm{D} \mathcal{H}_{n}-\mathrm{SMB}$ for $n=1,2,3$ and 4 displayed in Fig. 4 . One sees in Fig. 4(a) and 4(c) that the input singularity point corresponding to $x_{s}=0$ is doubled for any $\alpha \in(0, \pi / 2]$. However, the input singularities located in other points $x_{s} \neq 0$ are doubled only in the region 


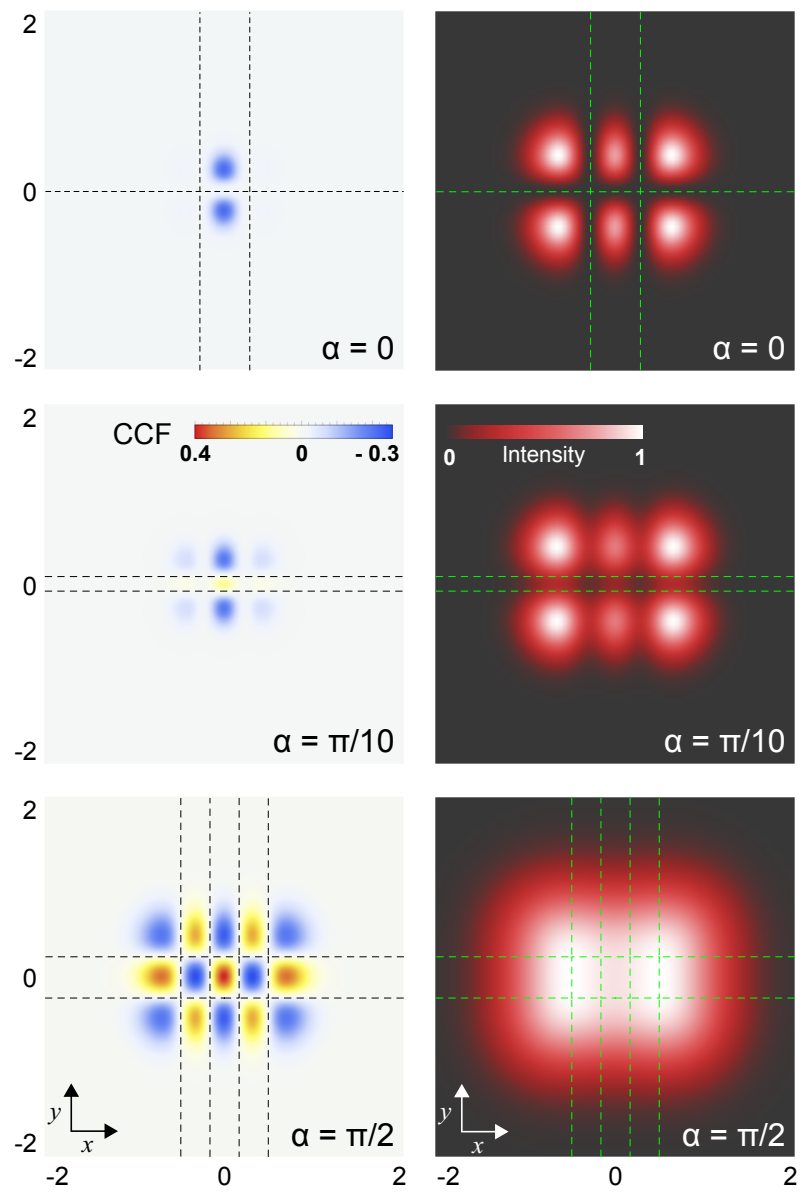

Fig. 3. (Color online) Evolution of the CCF (first column) and the intensity distribution (second column) during propagation $(\alpha)$ for the $2 \mathrm{D}$ SMBs associated with HG mode $\mathcal{H}_{2}(x) \mathcal{H}_{1}(y)$ in the input plane $(\alpha=0)$, Fresnel $(\alpha=\pi / 10)$, and Fraunhofer $(\alpha=\pi / 2)$ regions. Dashed lines indicate the position of CCF singularities: $\Gamma_{\mathcal{H}_{m} \mathcal{H}_{n}}\left(\mathbf{r},-\mathbf{r} ; \mathbf{T}_{\alpha}\right)=0$.
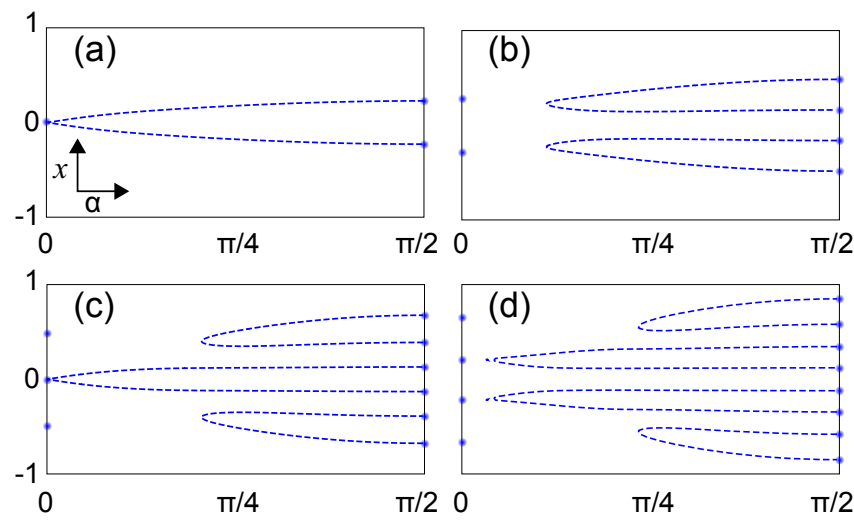

Fig. 4. (Color online) Evolution of the CCF singularity points during propagation $(\alpha)$ for the 1D SMBs associated with HG mode $\mathcal{H}_{1}, \mathcal{H}_{2}, \mathcal{H}_{3}$, and $\mathcal{H}_{4}$, presented in (a), (b), (c) and (d) respectively. Blue points at $\alpha=0$ and $\alpha=\pi / 2$ indicate the position of the CCF singularities in the input plane and far field, correspondingly. $\alpha \in\left(\alpha_{s}, \pi / 2\right]$, see Fig. 4(b), 4(c) and 4(d). The larger the $\left|x_{s}\right|$ the larger the $\alpha_{s}$ for a fixed value of $\rho$. Note that the increase of $\rho$ yields to the decrease of the distance $\left(\alpha_{S}\right)$ of singularities reappearance. We conclude that the formation of CCF singularity requires a certain degree of coherence around $x_{s}$ which is increased during beam propagation (see Fig. 2(a) and 2(b) and the first column of Fig. 3). The always higher degree of coherence around $x_{s}=0$ explains immediate doubling of this singularity in the input plane for $n=1$ and 3. These results demonstrate that the CCF has a memory about its input singularities, coinciding with the intensity zero points, which are finally revealed in far field in the form of the doubled singularity points. Thus the $2 \mathrm{D} \mathcal{H}_{m} \mathcal{H}_{n}-\mathrm{SMB}$ has in far field $2 m$ vertical and $2 n$ horizontal CCF singularity lines. As it has been previously mentioned, the CCF singularities of the SMB-LG in far field were studied in [7]. In particular, $2 p+|l|$ CCF ring singularities (where $p$ and $l$ are the radial and azimuthal mode indices of a LG mode) were observed. By comparing the results obtained for the HG- and LG-SMBs it follows that the number of CCF singularity curves in far field is related to the Gouy phase of the coherent mode defined by the index sum $k$ equals to $m+n$ and $2 p+|l|$, respectively. In the case of the closed curves (rings) the number is $k$ while for the open curves (lines) it is $2 k$.

The proposed analysis of the CCF singularity evolution is extendable for other SMBs associated with stable modes. The close relation between the shape, position and number of CCF singularity curves and the mode opens up promising perspectives for designing new information encoding protocols in free-space communications based upon partially coherent light. For the experimental observation of CCF singularities a simple interferometric setup as the one applied in [5, 7] can be used.

Spanish Ministerio de Economía y Competitividad is acknowledged for funding the project TEC2014-57394-P and FIS201346475-C3-1-P.

\section{REFERENCES}

1. M. Soskin and M. Vasnetsov, Progress in Optics 42, 219 (2001).

2. F. Gori, M. Santarsiero, R. Borghia, and S. Vicalvi, J. Mod. Opt. 45, 539 (1998).

3. G. Gbur and T. D. Visser, Opt. Commun. 222, 117 (2003).

4. G. V. Bogatyryova, C. V. Felde, P. V. Polyanskii, S. A. Ponomarenko, M. S. Soskin, and E. Wolf, Partially coherent vortex beams with a separable phase, Opt. Letts. 28, 878-880 (2003).

5. D. M. Palacios, I. D. Maleev, A. S. Marathay, and J. G. A. Swartzlander, Phys. Rev. Lett. 92, 143905 (2004).

6. I. D. Maleev, D. M. Palacios, A. S. Marathay, and J. G. A. Swartzlander, J. Opt. Soc. Am. B 21, 1895 (2004).

7. Y. Yang, M. Chen, M. Mazilu, A. Mourka, Y.-D. Liu, and K. Dholakia, New J. Phys. 15, 113053 (2013).

8. M. Born and E. Wolf, Principles of Optics (Cambridge University Press, UK, 2006).

9. A. C. Schell, "The multiple plate antenna", Ph.D. thesis, Massachusetts Institute of Technology (1961).

10. M. A. Alonso, Adv. Opt. Photon. 3, 272 (2011).

11. T. Alieva, J. A. Rodrigo, A. Cámara, and E. Abramochkin, J. Opt. Soc. Am. A 30, 2237 (2013).

12. H. M. Ozaktas, Z. Zalevsky, and M. A. Kutay, The Fractional Fourier Transform with Applications in Optics and Signal Processing (John Wiley\&Sons, NY, USA, 2001).

13. T. Alieva, Advances in Information Optics and Photonics ICO International Trends in Optics: Vol. VI (SPIE Press, USA, ISBN 9780819472342, 2008), chap. First-Order Optical Systems for Information Processing, pp. 1-26.

14. J. A. Rodrigo, T. Alieva, and M. L. Calvo, Opt. Express 17, 4976 (2009).

15. R. Simon and G. S. Agarwal, Optics Letters 25, 1313 (2000). 


\section{REFERENCES}

1. M. Soskin and M. Vasnetsov, "Singular optics," Progress in Optics 42, 219-2 (2001).

2. F. Gori, M. Santarsiero, R. Borghi, and S. Vicalvi, "Partially coherent sources with helicoidal modes," J. Mod. Opt. 45, 539-554 (1998).

3. G. Gbur and T. D. Visser, "Coherence vortices in partially coherent beams," Opt. Commun. 222, 117-125 (2003).

4. G. V. Bogatyryova, C. V. Felde, P. V. Polyanskii, S. A. Ponomarenko, M. S. Soskin, and E. Wolf, "Partially coherent vortex beams with a separable phase," Opt. Letts. 28, 878-880 (2003).

5. D. M. Palacios, I. D. Maleev, A. S. Marathay, and J. G. A. Swartzlander, "Spatial correlation singularity of a vortex field," Phys. Rev. Lett. 92, 143905 (2004).

6. I. D. Maleev, D. M. Palacios, A. S. Marathay, and J. G. A. Swartzlander, "Spatial correlation vortices in partially coherent light: theory," J. Opt. Soc. Am. B 21, 1895-1900 (2004).

7. Y. Yang, M. Chen, M. Mazilu, A. Mourka, Y.-D. Liu, and K. Dholakia, "Effect of the radial and azimuthal mode indices of a partially coherent vortex field upon a spatial correlation singularity," New J. Phys. 15, 113053 (2013).

8. M. Born and E. Wolf, Principles of Optics (Cambridge University Press, UK, 2006).

9. A. C. Schell, "The multiple plate antenna," Ph.D. thesis, Massachusetts Institute of Technology (1961).

10. M. A. Alonso, "Wigner functions in optics: describing beams as ray bundles and pulses as particle ensembles," Adv. Opt. Photon. 3, 272365 (2011).

11. T. Alieva, J. A. Rodrigo, A. Cámara, and E. Abramochkin, "Partially coherent stable and spiral beams," J. Opt. Soc. Am. A 30, 2237-2243 (2013).

12. H. M. Ozaktas, Z. Zalevsky, and M. A. Kutay, The Fractional Fourier Transform with Applications in Optics and Signal Processing (John Wiley\&Sons, NY, USA, 2001).

13. T. Alieva, Advances in Information Optics and Photonics ICO International Trends in Optics: Vol. VI (SPIE Press, USA, ISBN 9780819472342, 2008), chap. First-Order Optical Systems for Information Processing, pp. 1-26.

14. J. A. Rodrigo, T. Alieva, and M. L. Calvo, "Programmable twodimensional optical fractional Fourier processor," Opt. Express 17, 4976-4983 (2009).

15. R. Simon and G. S. Agarwal, "Wigner representation of laguerregaussian beams," Optics Letters 25, 1313-1315 (2000). 